\title{
Review
}

\section{Aging and longevity genes}

\author{
S. Michal Jazwinski ${ }^{\bowtie}$ \\ Department of Biochemistry and Molecular Biology, Louisiana State University Health Sciences \\ Center, New Orleans, Louisiana 70112 U.S.A.
}

Received: 13 March, 2000

Key words: aging, longevity genes

\begin{abstract}
The genetics of aging has made substantial strides in the past decade. This progress has been confined primarily to model organisms, such as filamentous fungi, yeast, nematodes, fruit flies, and mice, in which some thirty-five genes that determine life span have been cloned. These genes encode a wide array of cellular functions, indicating that there must be multiple mechanisms of aging. Nevertheless, some generalizations are already beginning to emerge. It is now clear that there are at least four broad physiological processes that play a role in aging: metabolic control, resistance to stress, gene dysregulation, and genetic stability. The first two of these at least are common themes that connect aging in yeast, nematodes, and fruit flies, and this convergence extends to caloric restriction, which postpones senescence and increases life span in rodents. Many of the human homologs of the longevity genes found in model organisms have been identified. This will lead to their use as candidate human longevity genes in population genetic studies. The urgency for such studies is great: The population is graying, and this research holds the promise of improvement in the quality of the later years of life.
\end{abstract}

Why do we age? This question has fascinated and troubled the human race for generations. However, this may not be the best way to phrase our concern about longevity and aging. In fact, I will argue that the relevant query is: Why do we live as long as we do? This subtle change in emphasis conceals a powerful in- sight into the aging process. It shifts the focus from a process that has evolved to serve a purpose to one that has escaped the force of natural selection [1]. It also argues forcefully against a program of aging, especially a genetic program in which each step is dependent on completion of the previous one in the se-

\footnotetext{
Research in my laboratory was supported by grants from the National Institute on Aging of the National Institutes of Health (U.S.P.H.S.).

${ }^{\bowtie}$ Send correspondence to: Dr. S. Michal Jazwinski, Department of Biochemistry and Molecular Biology, Louisiana State University Health Sciences Center, 1901 Perdido Street, Box P7-2, New Orleans, Louisiana 70112, U.S.A.; Phone/Fax 504568 4725; e-mail sjazwi@lsumc.edu
} 
quence. This argument against a genetic program of organismal aging is not inconsistent with programmed cell death or apoptosis, because cell death can be adaptive for the metazoan organism. After all, the organism is the object of natural selection, whose force is filtered, as it were, before impinging on the cells that constitute it. The foregoing does not in any way suggest that genes are not important in the biological aging process, in the sense that it is the genetic constitution that determines life span. Thus, the focal point moves from genes of aging to longevity assurance genes, which help determine the characteristic life span of the organism.

There have been many approaches to the study of aging. Through much of the past fifty years, much effort has been devoted to the description of biological aging as it takes place in mammals, particularly rodents and man. This description includes all levels of biological organization, from the molecular to the organismal, and it even reaches the supraorganismal in demography. This cataloging endeavor has spawned many theories of biological aging, encompassing virtually every level of description [2]. However, the past ten years have seen the rise of genetic analyses of aging, and, with it, the beginnings of an understanding of molecular mechanisms, pathways, and physiological processes important for longevity [3].

The emphasis of this article on genetics is not intended to belittle the importance of the environment in aging. Indeed, any phenotype, including aging, plays itself out through an interaction of genes, or more correctly the genotype, with the environment. There is also a probabilistic component to aging. This is evident from the fact that even under constant conditions members of a genetically homogeneous group of individuals do not die all in tandem. Thus, there exists an epigenetic stratification of such an aging population, the source of which has been modeled mathematically as random change [4]. This, however, lies beyond the scope of this presentation.

\section{WHAT IS AGING?}

Before we turn to a discussion of longevity genes, we must define the matter of the subject with which we are dealing. This is not a trivial task. Aging is not easy to define even though we know it when we see it. We are faced with a sliding scale of phenotypic characteristics encompassing profound decline and "successful aging". Successful aging is characterized by the avoidance of disease, the maintenance of high cognitive and physical function, and an engagement with life [5]. This characterization brings us closer to a definition of the biological aging process; however, it is evident that successful aging goes beyond biology per se. Indeed, it has been found that, in addition to physical exercise, social activity and productive pursuits contribute to what can be termed successful aging [6]. Clearly, the latter two enter the realm of the psychosocial. A useful definition of aging holds that it is a progressive decline in the ability to withstand stress, damage, and disease, and that it is characterized by an increase in the incidence of degenerative and neoplastic disorders.

A distinction is often made between the intrinsic aging process and age-related disease. (One extreme view has it that there is no intrinsic aging process, but only disease). This distinction has some important consequences; therefore, it is worth pursuing. To bring the difference between aging and disease into closer focus, it is worth considering the following scenario. An elderly man does not see a rock in his path, stumbles over it, loses his balance, and falls. His eyesight isn't what it used to be. More importantly, skeletal muscle atrophy has weakened his legs preventing easy recovery from the faulty footing. The fall results in a hip fracture; perhaps, there was an underlying osteoporotic process. In any case, he ends up a hip-replacement patient in the hospital. Healing slowly, his residence there is extended. His immunity not be- 
ing what it used to be, he contracts pneumonia and dies. The attending physician lists the cause of death as pneumonia. In reality, it could easily be argued that its ultimate cause was skeletal muscle atrophy. The disease was pneumonia, but the aging process included skeletal muscle atrophy and other functional decline.

Aging is not a disease, from the foregoing discussion. However, aging can predispose to disease. This is not surprising if we consider the functional decline that is the essence of the intrinsic aging process. Indeed, aging predisposes to a variety of diseases. Turning this around, we come to the conclusion that alleviation of some of the functional deficits of aging should reduce the incidence of many diseases and disorders. Thus, we can make major inroads in many age-related diseases and disorders by amelioration of aging, rather than taking the piecemeal approach of curing them one by one. This has obvious practical significance.

\section{GENETIC MODEL SYSTEMS}

Human genetics is the archival analysis of chance encounters. This designation contains the gist of one of the two problems in studying periment. For these reasons, we have turned to model organisms to study aging. Conservation of basic biological processes throughout phylogeny makes this approach rational. Furthermore, about one-fifth of human disease genes that have been positionally cloned possess yeast homologs, reflecting a remarkable degree of genetic conservation [7]. Many of these genes are interchangeable between the two species. The comparison between yeast and human is, of course, the most extreme example; we would expect even more similarity between humans and invertebrates.

The major genetic model systems used in aging research are the filamentous fungus Podospora anserina, bakers' yeast (Saccharomyces cerevisiae), the roundworm (Caenorhabditis elegans), the fruit fly (Drosophila melanogaster), and the mouse (Mus musculus). There are several genetic methodologies that have been applied to the study of aging in these organisms, although not necessarily all in each of them (Table 1). Mutagenesis involves the generation of mutants by physical or chemical treatment, followed by screening for individuals that possess the sought phenotypes. Molecular genetic strategies are similar except that they target known genetic loci that are considered candidates for genes involved in determining the phenotype of interest.

Table 1. Genetic model systems for the study of aging

\begin{tabular}{ll}
\hline Organism & Genetic methodology used \\
\hline Podospora anserina & Mutagenesis \\
Saccharomyces cerevisiae & Mutagenesis, molecular genetics \\
Caenorhabditis elegans & Mutagenesis, molecular genetics, QTL* analysis \\
Drosophila melanogaster & Mutagenesis, molecular genetics, QTL analysis, selective breeding \\
Mus musculus & Mutagenesis, molecular genetics, QTL analysis, selective breeding, \\
\hline
\end{tabular}

*quantitative trait loci

human aging. Genetically, humans are not very malleable both for practical and for ethical reasons. In addition, their life expectancy makes humans less attractive as the subject of aging research; it takes a lifetime to do an ex-
Quantitative trait loci (QTL) analysis encompasses a family of techniques that are used to zero in on the location of genes responsible for the variation in a particular phenotype. Selective breeding is used to progressively comb 
together the genetic variants in a population that are responsible for a particular trait by choosing for breeding in each generation individuals with the most extreme phenotypic expression. Associative genetics tests for differences in the frequency in a population of appearance of genetic variants in individuals displaying extreme values of the chosen phenotype.

These genetic methodologies have now identified some 35 cloned genes that determine longevity in the model organisms listed (Table 2). These genes encode a wide variety of highlight. I have used the term longevity genes in tabulating the progress of the genetics of aging in the model organisms. This emphasizes the fact that life span has been the phenotype analyzed in each case. The difficulties encountered with the definition of human aging are more acute when aging in these organisms is considered. This is because little has been done to describe the pathology of the aging organism. However, in some ways, this may be a blessing in disguise. The metric of life span keeps us on a firm footing, because the best predictor of mortality is life span it-

Table 2. Cloned longevity genes from model systems

\begin{tabular}{|c|c|c|c|c|}
\hline Podospora & Saccharomyces & Caenorhabditis & Drosophila & Mus \\
\hline \multirow[t]{18}{*}{ grisea } & $L A G 1$ & $d a f-2$ & $\operatorname{sod} 1$ & Prop-1 \\
\hline & $L A C 1$ & age-1/daf-23 & cat1 & $p 66^{s h c}$ \\
\hline & $R A S 1$ & $d a f-18$ & $m t h$ & \\
\hline & $R A S 2$ & $a k t-1 / a k t-2$ & & \\
\hline & PHB1 & $d a f-16$ & & \\
\hline & PHB2 & $d a f-12$ & & \\
\hline & $C D C 7$ & $c t l-1$ & & \\
\hline & $B U D 1$ & old-1 & & \\
\hline & $R T G 2$ & spe-26 & & \\
\hline & RPD3 & clk-1 & & \\
\hline & $H D A 1$ & mev-1 & & \\
\hline & SIR2 & & & \\
\hline & SIR4-42 & & & \\
\hline & UTH4 & & & \\
\hline & $Y G L 023$ & & & \\
\hline & $S G S 1$ & & & \\
\hline & $R A D 52$ & & & \\
\hline & $F O B 1$ & & & \\
\hline
\end{tabular}

proteins with a diversity of functions, suggesting the existence of many different mechanisms of aging.

At this point, little direct overlap between the functions identified in the different models is evident. However, as will be seen, there is considerable convergence of the physiological mechanisms of aging that these genes self. Thus, longevity becomes the tool, while health span is the target of investigation.

\section{Yeast}

The individual yeast cell buds a limited number of times, producing a daughter cell each time. The daughter has, in principle, the ca- 
pacity for a full replicative life span [8, 9]. Thus, the yeast population is immortal, even though the individual is mortal. The measure of the yeast life span is thus the number of divisions of the mother cell before it dies, and not chronological time. The genetic analysis of aging in yeast points to four broad physiological processes important for longevity. They are metabolic control, resistance to stress, gene dysregulation, and genetic stability. In addition, two molecular mechanisms of aging have been elucidated in this model system, on the basis of the genetic studies. Finally, the RAS2 gene has emerged as a homeostatic device in yeast longevity.

An intracellular signaling pathway from the mitochondrion to the nucleus, called the retrograde response, has been shown to determine yeast life span [10]. This pathway of interorganelle communication signals the functional status of the mitochondrion, leading to changes in the expression of nuclear genes that encode proteins that localize to the cytoplasm, the mitochondrion, and the peroxisome. The enzymatic changes that the retrograde response elicits represent a shift to the glyoxylate cycle to provide Krebs cycle intermediates, an induction of gluconeogenesis, and a change from the utilization of glucose to acetate. The latter translates into a shift to a carbon source of lower caloric content. These metabolic and enzymatic changes resemble those found in C. elegans mutants that display extended longevity. They are reminiscent of the metabolic changes found in fruit flies selected for extended life span, and they bear resemblance to some of the features of caloric restriction in rodents which results in the extension of life span. RAS2 modulates the retrograde response and the life extension it provides.

Resistance to ultraviolet radiation is associated with life span in yeast [11]. Similarly, resistance to oxidative stress is important for longevity [12]. Yeasts exposed to chronic bouts of sublethal heat stress show a curtailment of life span, which is more severe in the absence of $R A S 2$ [13]. This gene appears to be important in the downregulation of the response to heat once this stress is removed. Thus, it is as important for the cell to downregulate the stress response efficiently as it is for it to mount a robust response. Overexpression of RAS2 can completely abolish the deleterious effect on life span of chronic, sublethal heat stress. It acts through the adenylate cyclase signaling pathway in exerting this salutary effect. The importance of stress resistance for longevity is evidenced by the fact that the induction of thermotolerance early in life leads to a persistent, though not permanent, reduction in mortality rate resulting in extension of life span [14]. For this effect, both $R A S 1$ and $R A S 2$ are essential, as is HSP104.

One of the manifestations of aging is the loss of transcriptional silencing of genes located in the heterochromatic regions of the yeast genome near telomeres and at the silent mating type loci $[15,16]$. This gene dysregulation was suggested to be a possible cause of aging in yeast, and some evidence for its involvement in aging was forthcoming [3, 17]. The proposal that gene dysregulation plays a role in aging is now on a firm foundation. It has been shown that the yeast histone deacetylase genes RPD3 and HDA1 determine yeast longevity [18]. These genes not only affect the silencing status of the heterochromatic regions mentioned above, but they also modulate the silencing at the heterochromatic ribosomal DNA locus in yeast. In fact, their effects on longevity are associated with enhanced silencing of ribosomal DNA. The picture that is beginning to emerge is that aging results in dysregulation of the ribosomal DNA locus resulting in the excessive production of ribosomal RNA that is not balanced by the synthesis of ribosomal proteins. This would lead to the assembly of defective ribosomes. In fact, there is an increase in ribosomal RNA with age, coupled to a reduction in the efficiency of protein synthesis [19]. 
Another mechanism of aging involving the ribosomal DNA has been described. The tandem ribosomal DNA repeats lend themselves nicely to recombinational events that result in the excision of monomers which are known to generate circular species. These have been shown to accumulate with age, and their excessive accumulation can cause cell death [20]. It is not clear how this demise is actually brought about. This simple and attractive mechanism does not appear to play a dominant role in yeast aging. The retrograde response, whose induction extends life span, results in a massive increase in the ribosomal DNA circles. Thus, the circles are not sufficient to cause aging. They are also not necessary, because deletion of SIR2 which abrogates ribosomal DNA silencing and might be expected to result in circle production, shortens life span but does not generate circles [18]. This points to the role of SIR2 in silencing of ribosomal DNA in determining life span.

I have alluded to RAS2 as a homeostatic device in yeast longevity [21]. The source of this characterization is two-fold. First, there appears to be an optimal level of RAS2 expression associated with maximum longevity. This may be dependent on the genetic background, on environmental conditions, and on epigenetic exigencies. It should be noted that in the absence of overt stress it is an adenylate cyclase-independent pathway through which RAS2 acts to determine life span. Second, RAS2 modulates several processes that appear to play a role in yeast longevity. They include growth or metabolism, stress resistance, cell cycling, chromatin-dependent transcriptional silencing, and cellular spatial organization. I hypothesize that this gene also contributes to genetic stability. In this way, $R A S 2$ allocates cellular resources (energy and nutrients) to the competing demands made on them by these cellular processes. The only known signal to which it responds is nutritional status. It must maintain a balance in the face of shifting requirements. In a sense, this is the disposable soma theory of aging reduced to the molecular level. The homeostat in both cases cannot afford to put all the eggs in one basket. In the case of the disposable soma, the balance is struck between somatic maintenance and reproduction.

\section{C. elegans}

A signal transduction pathway that determines the life span of the worm has been elaborated [22]. At its head is the insulin receptor-like gene $d a f-2$, whose ligand is not known at present. The signal this receptor receives is transduced to the phosphatidylinositol-3-hydroxyl kinase gene age-1/daf-23, which in turn transmits the signal to akt-1/akt-2 (protein kinase B) [23]. This signal is modulated by daf-18, a phosphatidylinositol phosphatase gene [24]. The downstream effector of this pathway is a forkhead transcription factor encoded by daf-16. Interestingly, HNF-3 a forkhead transcription factor involved in eliciting the metabolic effects of insulin is downregulated by the insulin pathway [25], much like DAF-16 is downregulated by the DAF-2 pathway. The elimination of the DAF-2 signal extends life span. It also results in profound metabolic changes, including the accumulation of lipid and glycogen. The enzymatic changes that occur resemble those found in the retrograde response, including a shift to the glyoxylate cycle [26].

One other daf gene impinges on the pathway described above. This gene, daf-12, encodes a member of the steroid receptor superfamily. Mutants in this gene act synergistically with $d a f-2$ mutants in extending life span [27]. DAF-12 also appears to be involved in the transmisson of signals from germ cells to DAF-16 [28]. DAF-2 is the recipient of signals from the somatic gonad [28]. All of this makes for a rather intricate network of signaling that determines life span of the adult worm.

The downstream targets of this DAF pathway for determining life span are not known, except for one. This appears to be a cytoplas- 
mic catalase encoded by $c t l-1$ [29]. It is therefore not surprising that this pathway not only plays a role in determining life span, but that it also is important in stress resistance. Indeed, the pathway specifies resistance to a multiplicity of stressors, including heat, ultraviolet radiation, and oxidative stress [30].

Another set of genes also affects nematode aging. This set of genes is typified by $c l k-1$ [31]. There is some controversy whether or not this gene falls into the same category as the $d a f$ genes in having $d a f-16$ as a downstream effector and in specifying stress resistance. CLK-1 protein is homologous to the yeast Coq7 protein, which is involved in ubiquinone biosynthesis. This may not be the only function of CLK-1 in the nematode; it may not even be the essential function. Clearly, there are changes in metabolism, including a slowing of its rate, in clk-1 mutants. It has been suggested that in this mutant a signal is sent from the mitochondrion to the nucleus that impacts longevity [32]. This is reminiscent of the retrograde response.

\section{D. melanogaster}

Until recently, the major impact of genetics in aging research in Drosophila has been the selection of lines displaying extended longevity [33, 34]. Fruit flies thus selected show a greater metabolic capacity. They are also resistant to heat, desiccation, and ethanol vapors, and they have higher activities of several antioxidative enzymes. They are more efficient in their utilization of nutrients, and they have enhanced storage of lipid and glycogen. Many of these features bear similarity to ones found in yeast and worms that have an extended life span [35].

Direct evidence for the significance of oxidative stress in fly aging has been obtained through the generation of transgenic animals expressing either copper/zinc superoxide dismutase, catalase, or both. The transgenic flies lived longer while suffering less oxidative damage [36, 37]. In fact, expression of the dismutase in motorneurons alone was sufficient to increase life span, pointing to the importance of this tissue for fly longevity [38].

The mutagenesis approach has been successful in Drosophila more recently. A gene encoding a G-protein coupled transmembrane receptor called methuselah was identified in a screen for mutants showing increased longevity [39]. Coincident with the increased longevity was an enhanced resistance to heat, starvation, and oxidative stress. It has not been determined whether the mutant alters metabolic capacity.

\section{Mouse}

The oldest method for extending rodent life span is the reduction of caloric intake [40]. Although this is not a genetic approach, it has recently been subjected to gene expression profiling [41]. Two aspects of the global patterns of gene expression deserve some comment. First, they confirm earlier studies indicating the altered metabolism of the calorie restricted animals. Second, the altered gene expression portends the kind of metabolic changes that occur in yeasts in which the retrograde response has been induced. Calorie restricted rodents are resistant to stress, show a postponement of many of the functional deficits associated with aging, exhibit a delayed appearance of various age-related pathologies, such as cancer, and have an increased lifetime metabolic capacity [40]. (Induction of the retrograde response renders yeast more resistant to stress, as well). It has been proposed that calorie restricted animals display these features because of a change in the way that glucose is metabolized, in essence more effectively [40].

Two genetic interventions have been shown to extend the life span of the mouse. The Ames dwarf mouse (Prop-1) has a deficit in the development of the pituitary that results in a marked reduction in growth hormone production. This mouse is small, as the name indicates, but it has an enhanced longevity [42]. 
There must be metabolic consequences to this mutation, but the analysis has not yet been reported. More recently, a mutation in the gene encoding $\mathrm{p} 66^{\text {shc }}$, which is involved in signal transduction involving c-H-ras1, has been found to increase life span in the mouse [43]. The consequences are a marked reduction in apoptosis and resistance to oxidative stress. No other phenotypic changes have been described; there is apparently no change in size of the animal.

Evidence for epigenetic changes during aging, similar to those discussed in yeast, has been found in the mouse. This involves the stable somatic inactivation on translocation to the $\mathrm{X}$ chromosome of autosomal genes that determine coat color. The epigenetic control of the inactivation decreased with age, but the gene expression was random in time and location [44]. This sort of random change with age has been discussed here earlier. It is the kind of change that could give rise to epigenetic stratification of individuals in a population, resulting in the variance of life spans about the mean in an inbred population maintained under constant conditions. One might imagine that the frequency, though not the probabilistic nature, of such epigenetic changes might be under genetic control. This is in fact the case. A putative genetic locus has been identified in the mouse. This locus affects the variance of life spans of an aging cohort about the mean [45]. This locus was demonstrated in recombinant inbred lines; it affects the variance but not the mean life span.

\section{RAMIFICATIONS FOR HUMAN AGING}

\section{Life maintenance reserve}

Why do we live as long as we do? It's the longevity genes that are responsible. They help to specify the metabolic capacity and the stress responses that interplay with epigenetic factors, such as the environment during development, to establish the life maintenance re- serve [3]. This is not a physical entity, but rather an abstract concept. This life maintenance reserve establishes our functional capacity that allows us to withstand the assaults of the environment and epigenetic change to retain homeostasis. This capacity is not unlimited, and the probability that it will be overwhelmed increases with time, especially because it does not remain intact but is constantly changing. The life maintenance reserve determines why we live as long as we do. It is not the derivative of an aging program that is specified by aging genes.

\section{Towards human longevity genes}

With the identification, cloning, and characterization of longevity genes from model organisms, the next step in the hunt for human longevity genes can be contemplated. Human homologs of the genes from the model organisms must be cloned. This step has already been taken in several instances. One example is the human homolog of yeast LAG1 [46]. The human gene is located at $19 \mathrm{p} 12$. It can replace the yeast gene where it performs longevity gene function. The human LAG1 and other human homologs of the longevity genes from model organisms can be considered candidates for human longevity genes. This identity must, however, be verified by demonstrating that the candidate gene indeed performs this function in humans. This task can be approached genetically.

Linkage analysis has been used to map human genes associated with a particular trait. Usually, disease genes that result from mutations at a single locus have been hunted down in this manner, followed by positional cloning. This is a methodology that is nearly impossible to employ in the case of a complex trait such as aging. Aging is particularly difficult to address in this manner because it is not very likely that one would identify parents of individuals who are very long-lived. Longevity, after all, would again be the most direct phenotype to consider. 
Fortunately, there are other approaches that can be utilized. They include allele sharing, using affected siblings, and associative genetics, a population based approach [47]. The latter methodology has been useful in the identification of risk factors in complex genetic disorders. It has already been employed in aging studies. The most universal finding in this domain is that the $A P O E$ gene is associated with human longevity [48]. We have begun the Louisiana Centenarian Study to test the candidate genes developed in the yeast system for longevity function in humans, using first an associative genetic approach. There may be something special about extreme old age. There is a hint that mortality rate plateaus at older ages in humans [49]. There is no doubt that this happens for older yeasts [4]. It is obvious that it would be worthwhile to determine the nature of the factors that lead to such a plateau, because this could provide a means to improve human function in old age.

\section{Demographics}

The elderly population is growing rapidly in relative numbers in the developed world. Indeed, those 85 and older are increasing at a rate 6 -times faster than the general population, while for those 100 and older the rate is 10-times faster than the population at large. This has led many to project that the social security and medicare systems will be bankrupted early in this century. At least one way for preventing such an occurrence is to improve the quality of life in old age by helping people to remain functionally independent. This would reduce the strain on our social fabric, and help us enjoy the later years of life. The goal of aging research then is for people 'to die young at an old age'.

I would like to thank the organizers of the symposium for inviting me to make these opening comments.

\section{R E F E R E N C E S}

1. Rose, M.R. (1991) Evolutionary Biology of Aging. Oxford University Press, New York.

2. Warner, H.R., Butler, R.N., Sprott, R.L. \& Schneider, E.L. (1987) Modern Biological Theories of Aging. Raven Press, New York.

3. Jazwinski, S.M. (1996) Longevity, genes, and aging. Science 273, 54-59.

4. Jazwinski, S.M., Kim, S., Lai, C.-Y. \& Benguria, A. (1998) Epigenetic stratification: The role of individual change in the biological aging process. Exp. Gerontol. 33, 571-580.

5. Rowe, J.W. \& Kahn, R.L. (1998) Successful Aging. Pantheon Books, New York.

6. Glass, T.A., de Leon, C.M., Marottoli, R.A. \& Berkman, L.F. (1999) Population based study of social and productive activities as predictors of survival among elderly Americans. Brit. Med. J. 319, 478-483.

7. Bassett, D.E., Boguski, M.S., Spencer, F., Reeves, R., Kim, S-h., Weaver, T. \& Hieter, P. (1997) Genome cross-referencing and XREFdb: Implications for the identification and analysis of genes mutated in human disease. Nature Genet. 15, 339-344.

8. Mortimer, R.K. \& Johnston, J.R. (1959) Life span of individual yeast cells. Nature 183, 1751-1752.

9. Muller, I., Zimmermann, M., Becker, D. \& Flomer, M. (1980) Calendar life span versus budding life span of Saccharomyces cerevisiae. Mech. Ageing Dev. 12, 47-52.

10. Kirchman, P.A., Kim, S., Lai, C.-Y. \& Jazwinski, S.M. (1999) Interorganelle signaling is a determinant of longevity in Saccharomyces cerevisiae. Genetics 152, 179-190.

11. Kale, S.P. \& Jazwinski, S.M. (1996) Differential response to UV stress and DNA damage during the yeast replicative life span. Dev. Genet. 18, 154-160. 
12. Wawryn, J., Krzepilko, A., Myszka, A. \& Bilinski, T. (1999) Deficiency in superoxide dismutases shortens life span of yeast cells. Acta Biochim. Polon. 46, 249-253.

13. Shama, S., Kirchman, P.A., Jiang, J.C. \& Jazwinski, S.M. (1998) Role of RAS2 in recovery from chronic stress: Effect on yeast life span. Exp. Cell Res. 245, 368-378.

14. Shama, S., Lai, C.-Y., Antoniazzi, J.M., Jiang, J.C. \& Jazwinski, S.M. (1998) Heat stress-induced life span extension in yeast. Exp. Cell Res. 245, 379-388.

15. Kim, S., Villeponteau, B. \& Jazwinski, S.M. (1996) Effect of replicative age on transcriptional silencing near telomeres in Saccharomyces cerevisiae. Biochem. Biophys. Res. Commun. 219, 370-376.

16. Smeal, T., Claus, J., Kennedy, B., Cole, F. \& Guarente, L. (1996) Loss of transcriptional silencing causes sterility in old mother cells of $S$. cerevisiae. Cell 84, 633-642.

17. Jazwinski, S.M. (1990) Aging and senescence of the budding yeast Saccharomyces cerevisiae. Mol. Microbiol. 4, 337-343.

18. Kim, S., Benguria, A., Lai, C.-Y. \& Jazwinski, S.M. (1999) Modulation of life-span by histone deacetylase genes in Saccharomyces cerevisiae. Mol. Biol. Cell 10, 3125-3136.

19. Motizuki, M. \& Tsurugi, K. (1992) The effect of aging on protein synthesis in the yeast Saccharomyces cerevisiae. Mech. Ageing Dev. 64, 235-245.

20. Sinclair, D.A. \& Guarente, L. (1997) Extrachromosomal rDNA circles - A cause of aging in yeast. Cell 91, 1033-1042.

21. Jazwinski, S.M. (1999) The $R A S$ genes: A homeostatic device in Saccharomyces cerevisiae longevity. Neurobiol. Aging 20, 471478.

22. Tissenbaum, H.A. \& Ruvkun, G. (1998) An insulin-like signaling pathway affects both lon- gevity and reproduction in Caenorhabditis elegans. Genetics 148, 703-717.

23. Paradis, S. \& Ruvkun, G. (1998) Caenorhabditis elegans Akt/PKB transduces insulin-like receptor signals from AGE-1 PI3 kinase to the DAF-16 transcription factor. Genes Dev. 12, 2488-2498.

24. Ogg, S. \& Ruvkun, G. (1998) The C. elegans PTEN homolog, DAF-18, acts in the insulin receptor-like metabolic signaling pathway. Mol. Cell 2, 887-893.

25. O’Brien, R.M., Noisin, E.L., Suwanichkul, A., Yamasaki, T., Lucas, P.C., Wang, J.C., Powell, D.R. \& Granner, D.K. (1995) Hepatic nuclear factor-3 and hormone-regulated expression of the phosphoenolpyruvate carboxykinase and insulin-like growth factor-binding protein 1 genes. Mol. Cell. Biol. 15, 1747-1758.

26. Vanfleteren, J.R. \& De Vreese, A. (1995) The gerontogenes age-1 and daf-2 determine metabolic rate potential in aging Caenorhabditis elegans. FASEB J. 9, 1355-1361.

27. Larsen, P.L., Albert, P.S. \& Riddle, D.L. (1995) Genes that regulate both development and longevity in Caenorhabditis elegans. Genetics 139, 1567-1583.

28. Hsin, H. \& Kenyon, C. (1999) Signals from the reproductive system regulate the lifespan of $C$. elegans. Nature 399, 362-366.

29. Taub, J., Lau, J.K., Ma, C., Hahn, J.H., Hoque, R., Rothblatt, J. \& Chalfie, M. (1999) A cytosolic catalase is needed to extend adult lifespan in C. elegans daf-C and clk-1 mutants. Nature 399, 162-166.

30. Murakami, S. \& Johnson, T.E. (1996) A genetic pathway conferring life extension and resistance to UV stress in Caenorhabditis elegans. Genetics 143, 1207-1218.

31. Ewbank, J.J., Bames, T.M., Lakowski, B., Lussier, M., Bussey, H. \& Hekimi, S. (1997) Structural and functional conservation of the 
Caenorhabditis elegans timing gene clk-1. Science 275, 980-983.

32. Felkai, S., Ewbank, J.J., Lemieux, J., Labbe, J.-C., Brown, G.G. \& Hekimi, S. (1999) CLK-1 controls respiration, behavior and aging in the nematode Caenorhabditis elegans. EMBO J. 18, 1783-1792.

33. Luckinbill, L.S., Arking, R., Clare, M.J., Cirocco, W.C. \& Buck, S.A. (1984) Selection for delayed senescence in Drosophila melanogaster. Evolution 38, 996-1003.

34. Rose, M.R. (1984) Laboratory evolution of postponed senescence in Drosophila melanogaster. Evolution 38, 1004-1010.

35. Jazwinski, S.M. (2000) Coordination of metabolic activity and stress resistance in yeast longevity; in The Molecular Genetics of Aging (Hekimi, S., ed.) pp. 21-44, Springer- Verlag, Berlin.

36. Orr, W.C. \& Sohal, R.S. (1994) Extension of life-span by overexpression of superoxide dismutase and catalase in Drosophila melanogaster. Science 263, 1128-1130.

37. Sun, J. \& Tower, J. (1999) FLP recombinasemediated induction of $\mathrm{Cu} / \mathrm{Zn}$-superoxide dismutase transgene expression can extend the life span of adult Drosophila melanogaster flies. Mol. Cell. Biol. 19, 216-228.

38. Parkes, T.L., Elia, A.J., Dickinson, D., Hilliker, A.J., Phillips, J.P. \& Boulianne, G.L. (1998) Extension of Drosophila lifespan by overexpression of human SOD1 in motorneurons. Nature Genet. 19, 171-174.

39.Lin, Y.-J., Seroude, L. \& Benzer, S. (1998) Extended life-span and stress resistance in the Drosophila mutant methuselah. Science $\mathbf{2 8 2}$, 943-946.

40. Masoro, E. (1995) Dietary restriction. Exp. Gerontol. 30, 291-298.
41. Lee, C.-K., Klopp, R.G., Weindruch, R. \& Prolla, T.A. (1999) Gene expression profile of aging and its retardation by caloric restriction. Science 285, 1390-1393.

42. Brown-Borg, H., Borg, K.E., Meliska, C.J. \& Bartke, A. (1996) Dwarf mice and the ageing process. Nature 384, 33.

43. Migliaccio, E., Giorgio, M., Mele, S., Pelicci, G., Reboldi, P., Pandolfi, P.P., Lanfrancone, L. \& Pelicci, P.G. (1999) The $\mathrm{p} 66^{\text {shc }}$ adaptor protein controls oxidative stress response and life span in mammals. Nature 402, 309-313.

44. Cattanach, B.L. (1974) Position effect variegation in the mouse. Genet. Res. 23, 291-306.

45. de Haan, G., Gelman, R., Watson, A., Yunis, E. \& Van Zant, G. (1998) A putative gene causes variability in lifespan among genotypically identical mice. Nature Genet. 19, $114-116$.

46.Jiang, J.C., Kirchman, P.A., Zagulski, M., Hunt, J. \& Jazwinski, S.M. (1998) Homologs of the yeast longevity gene LAG1 in Caenorhabditis elegans and human. Genome Res. 8, 1259-1272.

47. Lander, E.S. \& Schork, N.J. (1994) Genetic dissection of complex traits. Science 265, 2037-2048.

48. Schachter, F., Faure-Delanef, L., Guenot, F., Rouger, H., Froguel, P. Lesueur-Ginot, L. \& Cohen, D. (1994) Genetic associations with human longevity at the $A P O E$ and $A C E$ loci. Nature Genet. 6, 29-32.

49. Vaupel, J.W., Carey, J.R., Christensen, K., Johnson, T.E., Yashin, A.I., Holm, N.V., Iachine, I.A., Kannisto, V., Khazaeli, A.A., Liedo, P., Longo, V.D., Zeng, Y., Manton, K.G. \& Curtsinger, J.W. (1998) Biodemographic trajectories of longevity. Science 280, 855860. 